\title{
Technologies and Materials for Carbon Dioxide Capture
}

\section{${ }^{1}$ Indri Susanti}

${ }^{1}$ Department of Science Education, Faculty of Teacher Training and Education, Lamongan Islamic University. Jl. Veteran No.53A Lamongan, Indonesia Email: indri_susanti@unisla.ac.id

Article Info
Article History
Received: 06 September
2019
Revised: $15 \quad$ September
2019
Published: 30 September
2019
Keywords
Technology; Material; $\mathrm{CO}_{2}$
Adsorption

Sitasi: Susanti, Indri. (2019). Technologies and Materials for Carbon Dioxide Capture. Science Education and Application Journal (SEAJ). Vol. 1, No. 2: 84-97

\section{PENDAHULUAN (INTRODUCTION)}

Carbon dioxide is one of the triggers for the greenhouse effect and global warming. The concentration of $\mathrm{CO}_{2}$ gas in the atmosphere in 2014 reached $400 \mathrm{ppm}$, the amount of which was much greater during the pre-industrialization era $(\mathrm{Oh}, 2010)$. Increasing the concentration of $\mathrm{CO}_{2}$ continuously in the atmosphere will limit the ability of plants and seas to absorb $\mathrm{CO}_{2}$ gas, so that carbon dioxide gas will be trapped in the earth's atmosphere and cause global warming which is characterized by rising earth temperatures, shifting climate, and rising sea levels (Li, J.-R.dkk., 2011: Yang, H. dkk., 2008). These problems must be addressed immediately so that global warming can be reduced.

Some methods to reduce $\mathrm{CO}_{2}$ gas problems are separation technologies include air capture, CCUS ( $\mathrm{CO}_{2}$ Capture Utilization and Storage), and CCS (Carbondioxide Capture and Storage) technology. Technology with several systems, namely post-combution, precombustion and oxy-combustion (Figueroa, 2008). Post-combution systems can be done in various ways including absorption, adsorption, membrane, and cryogenic.

Description of the operation and explanation of some of these technologies will be discussed in this paper. The advantages and disadvantages of each technology will also increase the efficiency of technology that is promising for the future. The objectives of this paper is to review the technologies and materials for $\mathrm{CO}_{2}$ capture.

\section{METODE (METHODS)}

\section{Carbon Dioxide Separation Methods}

The problem of carbon dioxide can be overcome through carbon dioxide gas separation technology as shown in Table 1. 
Tabel 1. Gas Separation Technologies

\begin{tabular}{|c|c|c|}
\hline Tecnology & Explanation & Reference \\
\hline Air Capture & $\begin{array}{l}\text { - Capture } \mathrm{CO}_{2} \text { gas up to } 52 \% \\
\text { - The use of amines as gas binders is } \\
\text { considered unsafe }\end{array}$ & $\begin{array}{l}\text { Kintisch, E., (2014). Can } \\
\text { Sucking } \mathrm{CO}_{2} \text { Out of the } \\
\text { Atmosphere Really Work, } \\
\text { Columbia: MIT } \\
\text { Technology Review. }\end{array}$ \\
\hline $\begin{array}{c}\text { CCUS } \\
\left(\mathrm{CO}_{2} \text { Capture }\right. \\
\text { Utilization and } \\
\text { Storage })\end{array}$ & $\begin{array}{l}\text { - Inject } \mathrm{CO}_{2} \text { on the inside of an } \\
\text { underground adsorbent } \\
\text { - Dangerous when the gas pipe leaks } \\
\text { causing contamination of ground water } \\
\text { - Affects drinking water TDS }\end{array}$ & $\begin{array}{l}\text { Bielicki, J. M. dkk., } \\
\text { (2014). Causes and } \\
\text { financial consequences of } \\
\text { geologic CO2 storage } \\
\text { reservoir leakage and } \\
\text { interference with other } \\
\text { subsurface resources. } \\
\text { International Journal of } \\
\text { Greenhouse Gas Control, } \\
\text { 20.272-284. }\end{array}$ \\
\hline $\begin{array}{c}\mathrm{CCS} \\
\left(\mathrm{CO}_{2} \text { Capture }\right. \\
\text { and Storage })\end{array}$ & $\begin{array}{l}\text { - Separation of combustion } \mathrm{CO}_{2} \text { gas } \\
\text { - Derivative systems: pre-combustion, post } \\
\text { combustion and oxy fuel combustion. }\end{array}$ & $\begin{array}{l}\text { Bennaucer, K., Gielen, } \\
\text { D., Kerr, T. \& Tam, C., } \\
\text { (2008). CO2 Capture and } \\
\text { Storage. International } \\
\text { Energy Agency, p. } 15 .\end{array}$ \\
\hline
\end{tabular}

Based on Table 1, CCS technology was chosen and reviewed for carbon dioxide gas separation because this technology has low risk of danger and cost. The three systems derived from CCS technology are pre-combustion, post combustion and oxy fuel combustion was described in Table 2.

In pre-combustion capture, carbon and carbon dioxide in fossil fuels are separated before combustion. This fuel source is chemically converted into a stream of two gases, carbon dioxide and $\mathrm{CO}_{2}$. The process of Pre-combustion Capture ${ }^{[9]}$ is shown in Figure 1.

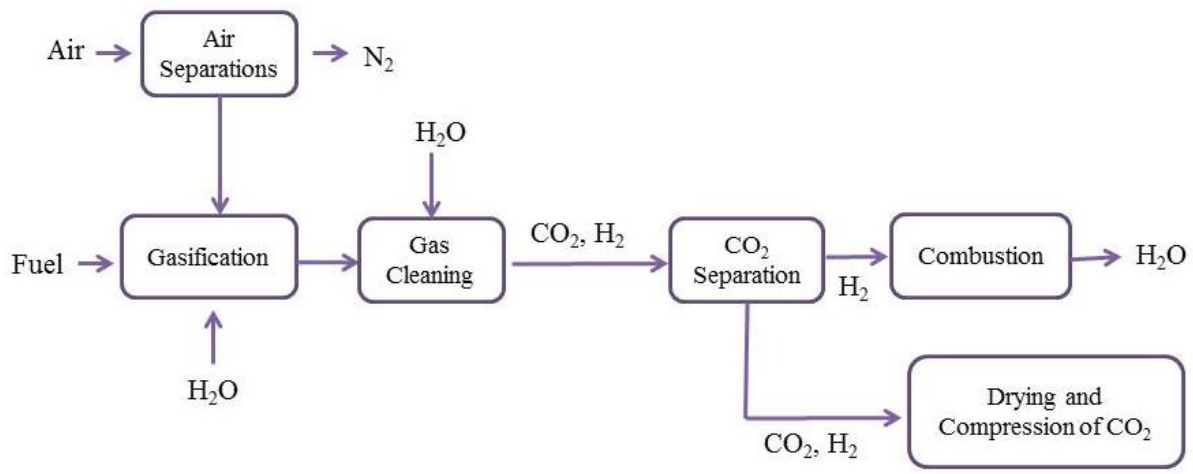

Figure 1. Flow chart of Pre-combustion Capture process

Tabel 2. $\mathrm{CO}_{2}$ Gas Separation Technologies

\begin{tabular}{|c|l|l|l|}
\hline $\begin{array}{c}\text { Technology of } \\
\mathbf{C O}_{2} \text { Sorption }\end{array}$ & \multicolumn{1}{|c|}{ Advantages } & \multicolumn{1}{c|}{ Disadvantages } & \multicolumn{2}{|c|}{ Reference } \\
\hline pre-combustion & $\begin{array}{l}\text { Produces synthetic gas } \\
\text { (syngas) rich in } \\
\text { hydrogen. }\end{array}$ & $\begin{array}{l}\text { Synthetic gases (syngas) } \\
\text { produced can cause } \\
\text { corrosion. }\end{array}$ & $\begin{array}{l}\text { Li, J.-R.dkk., } \\
\text { (2011). Carbon } \\
\text { dioxide capture- }\end{array}$ \\
\hline
\end{tabular}




\begin{tabular}{|c|c|c|c|}
\hline $\begin{array}{l}\text { Technology of } \\
\mathrm{CO}_{2} \text { Sorption }\end{array}$ & Advantages & Disadvantages & Reference \\
\hline & $\begin{array}{l}\text { Capable of capturing } \\
\text { almost } 91.6 \% \text { of } \mathrm{CO}_{2} \\
\text { emissions. }\end{array}$ & $\begin{array}{l}\text { The process is more } \\
\text { complicated and the cost } \\
\text { to build a system is very } \\
\text { expensive. }\end{array}$ & $\begin{array}{lr}\text { related gas } \\
\text { adsorption and } \\
\text { separation in } \\
\text { metal-organic } \\
\text { frameworks. } \\
\text { Coordination } \\
\text { Chemistry } \\
\text { Reviews, } \\
\text { 1791-1823. }\end{array}$ \\
\hline $\begin{array}{l}\text { oxy fuel } \\
\text { combustion }\end{array}$ & $\begin{array}{l}\text { The } \mathrm{CO}_{2} \text { separation } \\
\text { process is easier because } \\
\text { it uses methods such as } \\
\text { absorption, membrane, } \\
\text { cryogenic and } \\
\text { adsorption. } \\
\text { Cheaper operating costs }\end{array}$ & $\begin{array}{l}\text { Requires quite high } \\
\text { energy when using } \\
\text { cryogenic and membrane } \\
\text { methods }\end{array}$ & $\begin{array}{l}\text { Miller, B.G., } \\
\text { (2011) CO2 } \\
\text { Capture dan } \\
\text { Storage, In Clean } \\
\text { Coal Engineering } \\
\text { Technology, } \\
\text { Butterworth- } \\
\text { Heinemann, } \\
\text { Boston, 483-511 }\end{array}$ \\
\hline post combustion & $\begin{array}{l}\text { The results of the } \\
\text { exhaust gas in the form } \\
\text { of } \mathrm{CO}_{2} \text { and water }\left(\mathrm{H}_{2} \mathrm{O}\right) \\
\text { so no } \mathrm{CO}_{2} \text { separation } \\
\text { process is needed. } \\
\text { Capable of capturing } \\
92.6 \% \mathrm{CO}_{2} \text { emissions. }\end{array}$ & $\begin{array}{l}\text { Requires very high costs } \\
\text { during the initial air } \\
\text { separation process. } \\
\text { Requires up to } 98 \% \text { pure } \\
\text { oxygen so the operating } \\
\text { costs are very high. }\end{array}$ & $\begin{array}{l}\text { Figueroa J. D., } \\
\text { Fout T., Plasynski } \\
\text { S., Mc. Ilvried H. } \\
\text { dan Srivastava R. } \\
\text { D., (2008), } \\
\text { Advances in CO2 } \\
\text { capture } \\
\text { technology, The } \\
\text { U.S. Department } \\
\text { of Energy's } \\
\text { Carbon } \\
\text { Sequestration } \\
\text { Program, } \\
\text { International } \\
\text { Journal of } \\
\text { Greenhouse Gas } \\
\text { Control, 21. 9-20 }\end{array}$ \\
\hline
\end{tabular}

In Oxy-fuel Combustion is simpler than pre-combustion capture because the oxidation process uses oxygen so it only produces $\mathrm{CO}_{2}$ and $\mathrm{H}_{2} \mathrm{O}$. Because the fuel used in the combustion process only produces $\mathrm{CO}_{2}$ and $\mathrm{H}_{2} \mathrm{O}$, there is no need to separate the exhaust gas anymore, so that the $\mathrm{CO}_{2}$ gas produced from this process can be directly captured. The process of Oxy-fuel Combustion $^{[9]}$ is shown in Figure 2. 


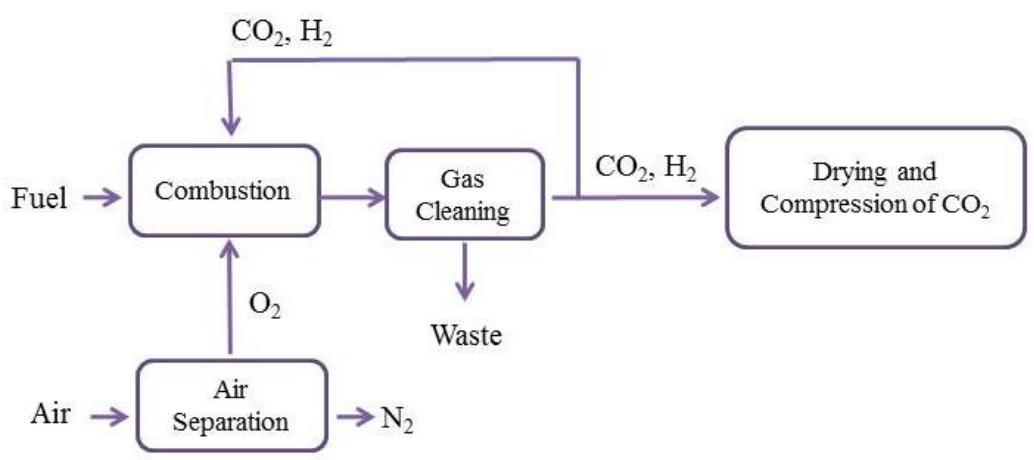

Figure 2. Flow chart of Oxy-fuel Combustion process

The last process is the post-combustion capture process. In this process, separating $\mathrm{CO}_{2}$ is easier because it uses methods such as adsorption, membrane, cryogenic, and adsorption. The process of Post-combustion Capture (Miller, 2011) is shown in Figure 3.

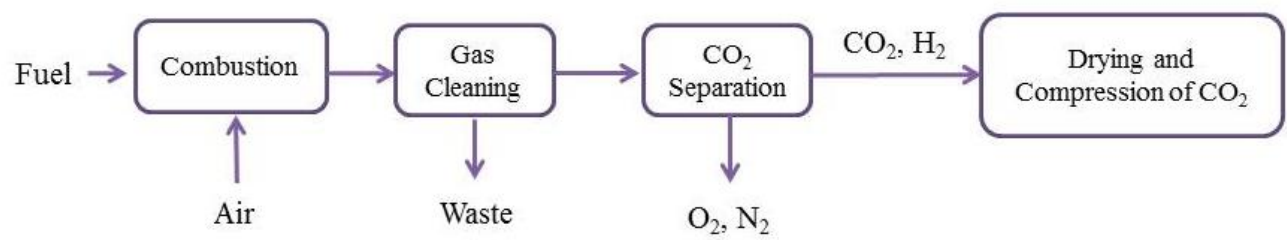

Figure 3. Flow chart of Post-combustion Capture process

The use of post-combustion capture technology has been more developed because $\mathrm{CO}_{2}$ separation methods are simpler and more efficient than the two processes above. The use of cryogenic and membrane methods requires high energy, but this can be avoided by choosing absorbs or adsorption methods to reduce operating costs. At present, the most commonly used carbon dioxide $\left(\mathrm{CO}_{2}\right)$ separation method in the post-combustion capture technology includes absorption, cryogenic, membranes, and adsorption. A comparison of the four carbon dioxide separation methods can be seen in Table 3 .

Tabel 3. $\mathrm{CO}_{2}$ Gas Separation System

\begin{tabular}{|c|c|c|c|c|c|}
\hline Methods & Explanation & $\begin{array}{c}\text { Energy } \\
\text { Needed } \\
(\mathrm{MJ} / \mathrm{Kg} \\
\left.\mathrm{CO}_{2}\right)\end{array}$ & $\begin{array}{l}\text { Pure } \\
\text { Limit }\end{array}$ & $\begin{array}{c}\text { The } \\
\text { unit of } \\
\text { time } \\
\text { reaches } \\
\text { the } \\
\text { pure } \\
\text { limit }\end{array}$ & Reference \\
\hline Absorption & $\begin{array}{l}\text { - The } \\
\text { interaction } \\
\text { between } \\
\text { adsorbent and } \\
\text { adsorbate } \\
\left(\mathrm{CO}_{2} \text { gas) is }\right. \\
\text { very strong so } \\
\text { it takes very } \\
\text { high energy to } \\
\text { separate the } \\
\text { adsorbent and }\end{array}$ & $0.5-4.5$ & 99 & Hour & $\begin{array}{l}\text { 1. Li, J.-R.dkk., (2011). } \\
\text { Carbon dioxide capture- } \\
\text { related gas adsorption and } \\
\text { separation in metal-organic } \\
\text { frameworks. Coordination } \\
\text { Chemistry Reviews, } 255 . \\
\text { 1791-1823. } \\
\text { 2. Pirngruber G. D., Guillou } \\
\text { F., Gomez A. dan Clausse } \\
\text { M., (2013), A theoretical } \\
\text { analysis of the energy }\end{array}$ \\
\hline
\end{tabular}




\begin{tabular}{|c|c|c|c|c|c|}
\hline Methods & Explanation & $\begin{array}{c}\text { Energy } \\
\text { Needed } \\
(\mathrm{MJ} / \mathrm{Kg} \\
\left.\mathrm{CO}_{2}\right)\end{array}$ & $\begin{array}{c}\text { Pure } \\
\text { Limit }\end{array}$ & $\begin{array}{l}\text { The } \\
\text { unit of } \\
\text { time } \\
\text { reaches } \\
\text { the } \\
\text { pure } \\
\text { limit }\end{array}$ & Reference \\
\hline & $\begin{array}{l}\text { adsorbate } \\
\text { again. } \\
\text { - The process is } \\
\text { more } \\
\text { complicated } \\
\text { because } \mathrm{CO}_{2} \\
\text { gas must be } \\
\text { converted to } \\
\text { solution using } \\
\text { chemical } \\
\text { solvents. }\end{array}$ & & & & $\begin{array}{l}\text { consumption of post- } \\
\text { combustion } \mathrm{CO}_{2} \text { capture } \\
\text { processes by temperature } \\
\text { swing adsorption using } \\
\text { solid sorbents", } \\
\text { International Journal of } \\
\text { Greenhouse Gas Control, } \\
\text { 14. 74-83 } \\
\text { 3. Smith, N. dkk., (2013), } \\
\text { Performance and Costs of } \\
\mathrm{CO}_{2} \text { Capture at Gas Fired } \\
\text { Power Plants. Energy } \\
\text { Procedia, 37. 2443-2452. }\end{array}$ \\
\hline Adsorbtion & 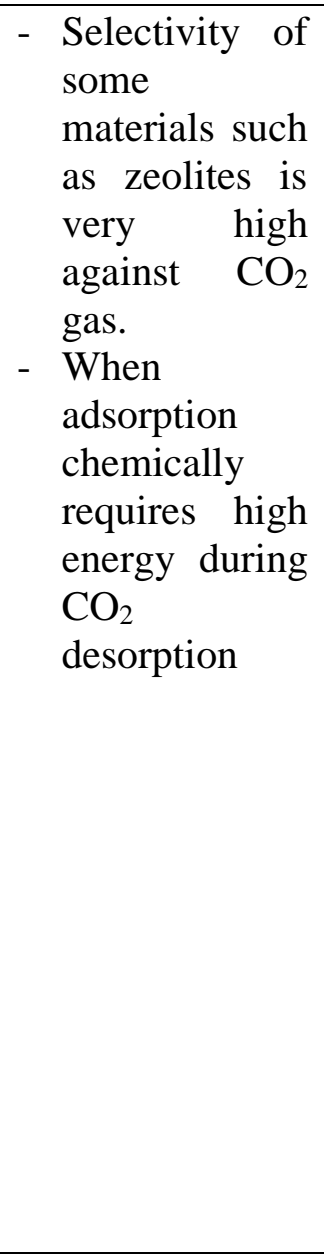 & $0.1-1$ & 95 & Minute & $\begin{array}{l}\text { 1. Li, J.-R.dkk., (2011). Carbon } \\
\text { dioxide capture-related gas } \\
\text { adsorption and separation in } \\
\text { metal-organic frameworks. } \\
\text { Coordination Chemistry } \\
\text { Reviews, 255. 1791-1823. } \\
\text { 2. Smith, N. dkk., (2013). } \\
\text { Performance and Costs of } \\
\mathrm{CO}_{2} \text { Capture at Gas Fired } \\
\text { Power Plants. Energy } \\
\text { Procedia, 37. 2443-2452. } \\
\text { 3. Cheung, O. dkk., (2013). } \\
\text { "Adsorption kinetics for } \\
\text { CO on highly selective } \\
\text { zeolites NaKA and nano- } \\
\text { NaKA". Ahallied Energy, } \\
\text { 112, hal. 1326-1336. } \\
\text { 4. Pham T. D., Xiong R., } \\
\text { Sdanler S. I. dan Lobo R. F., } \\
\text { (2014) Experimental dan } \\
\text { computational studies on the } \\
\text { adsorption of CO dan } \mathrm{N}_{2} \text { on } \\
\text { pure silica zeolites. } \\
\text { Microporous dan } \\
\text { Mesoporous Materials, 185. } \\
\text { 157-166. }\end{array}$ \\
\hline Membrane & $\begin{array}{l}\text { Selectivity for } \\
\mathrm{CO}_{2} \text { gas is still } \\
\text { relatively low } \\
\text { for }\end{array}$ & $\begin{array}{c}0.5- \\
16.56\end{array}$ & $<40$ & Minute & $\begin{array}{l}\text { 1. Li, J.-R.dkk., (2011). } \\
\text { Carbon dioxide capture- } \\
\text { related gas adsorption and } \\
\text { separation in metal-organic } \\
\text { frameworks. Coordination }\end{array}$ \\
\hline
\end{tabular}




\begin{tabular}{|c|c|c|c|c|c|}
\hline Methods & Explanation & $\begin{array}{c}\text { Energy } \\
\text { Needed } \\
(\mathrm{MJ} / \mathrm{Kg} \\
\left.\mathrm{CO}_{2}\right)\end{array}$ & $\begin{array}{l}\text { Pure } \\
\text { Limit }\end{array}$ & $\begin{array}{l}\text { The } \\
\text { unit of } \\
\text { time } \\
\text { reaches } \\
\text { the } \\
\text { pure } \\
\text { limit }\end{array}$ & Reference \\
\hline & $\begin{array}{l}\text { commercial } \\
\text { use } \\
\text { - Requires high } \\
\text { temperatures } \\
\text { to get high } \\
\mathrm{CO}_{2} \text { purity }\end{array}$ & & & & $\begin{array}{l}\text { Chemistry Reviews, } 255 . \\
\text { 1791-1823. } \\
\text { 2. Smith, N. dkk., (2013). } \\
\text { Performance and Costs of } \\
\mathrm{CO}_{2} \text { Capture at Gas Fired } \\
\text { Power Plants. Energy } \\
\text { Procedia, 37. 2443-2452 } \\
\text { 3. Kazama, S. dan Haraya, K., } \\
\text { (2013), Optimization of } \\
\mathrm{CO}_{2} \text { concentration captured } \\
\text { by membrane technology - } \\
\text { Possibility of reduction in } \\
\mathrm{CO}_{2} \text { capture energy dan } \\
\text { cost, Energy Procedia, } 37 . \\
969-975 .\end{array}$ \\
\hline Cryogenic & $\begin{array}{l}\text { - The } \\
\text { temperature } \\
\text { used is quite } \\
\text { low between } \\
103^{\circ} \mathrm{C}-122^{\circ} \mathrm{C} \\
\text { - Less efficient } \\
\text { because } \mathrm{CO}_{2} \\
\text { has to be } \\
\text { converted into } \\
\text { a liquid with a } \\
\text { very low } \\
\text { temperature }\end{array}$ & 0.4 & 99 & Hour & $\begin{array}{l}\text { 1. Li, J.-R.dkk., (2011). } \\
\text { Carbon dioxide capture- } \\
\text { related gas adsorption and } \\
\text { separation in metal-organic } \\
\text { frameworks. Coordination } \\
\text { Chemistry Reviews, } 255 . \\
\text { 1791-1823. } \\
\text { 2. Miller, B.G., (2011) CO } 2 \\
\text { Capture dan Storage, In } \\
\text { Clean Coal Engineering } \\
\text { Technology, Butterworth- } \\
\text { Heinemann, Boston, 483- } \\
\text { 511. } \\
\text { Smith, N. dkk., (2013). } \\
\text { Performance and Costs of } \\
\text { CO Capture at Gas Fired } \\
\text { Power Plants. Energy } \\
\text { Procedia, 37. 2443-2452. }\end{array}$ \\
\hline
\end{tabular}

The absorption and adsorption methods require lower operational costs compared to cryogenic or membrane methods. In the membrane method, selectivity to $\mathrm{CO}_{2}$ is still very low compared to adsorption, whereas the cryogenic method requires high energy to convert $\mathrm{CO}_{2(\mathrm{~g})}$ to $\mathrm{CO}_{2(1)}$ (Miller, 2011). Absorption methods generally use chemical solvents such as fluorinated solvents, ammonia solutions and ionic liquids ( $\mathrm{Li}, 2011)$ and require high energy because of the interaction between adsorbents (chemical solvents) and adsorbate $\left(\mathrm{CO}_{2}\right.$ gas) in the form of chemical bonds, making it very difficult to separate when adsorbent regeneration. While the adsorption method uses materials such as porous silica, carbon and zeolites $(\mathrm{Li}$, 
2011). Based on Table 3, the adsorption method has a lower pure limit than the absorption and cryogenic methods, but has a faster time to reach the pure limit that is in units of minutes. The pure limit is the maximum purity limit obtained by using the $\mathrm{CO}_{2}$ separation method. In addition, the adsorption method only requires lower energy when capturing $\mathrm{CO}_{2}$ than all methods, so it is more profitable.

\section{HASIL DAN PEMBAHASAN (RESULT AND DISCUSSION) \\ I. Materials for Carbon Dioxide Adsorption}

Some materials which have been developed for carbon dioxide adsorption are porous materials such as mesopore silica, zeolites, carbon, MOF (metal organic framework), and COF (covalent organic framework). Several studies showing the effect of adsorption type and pore diameter on the carbon dioxide adsorption capacity can be seen in Table 4.

Tabel 4. Materials for $\mathrm{CO}_{2}$ Capture

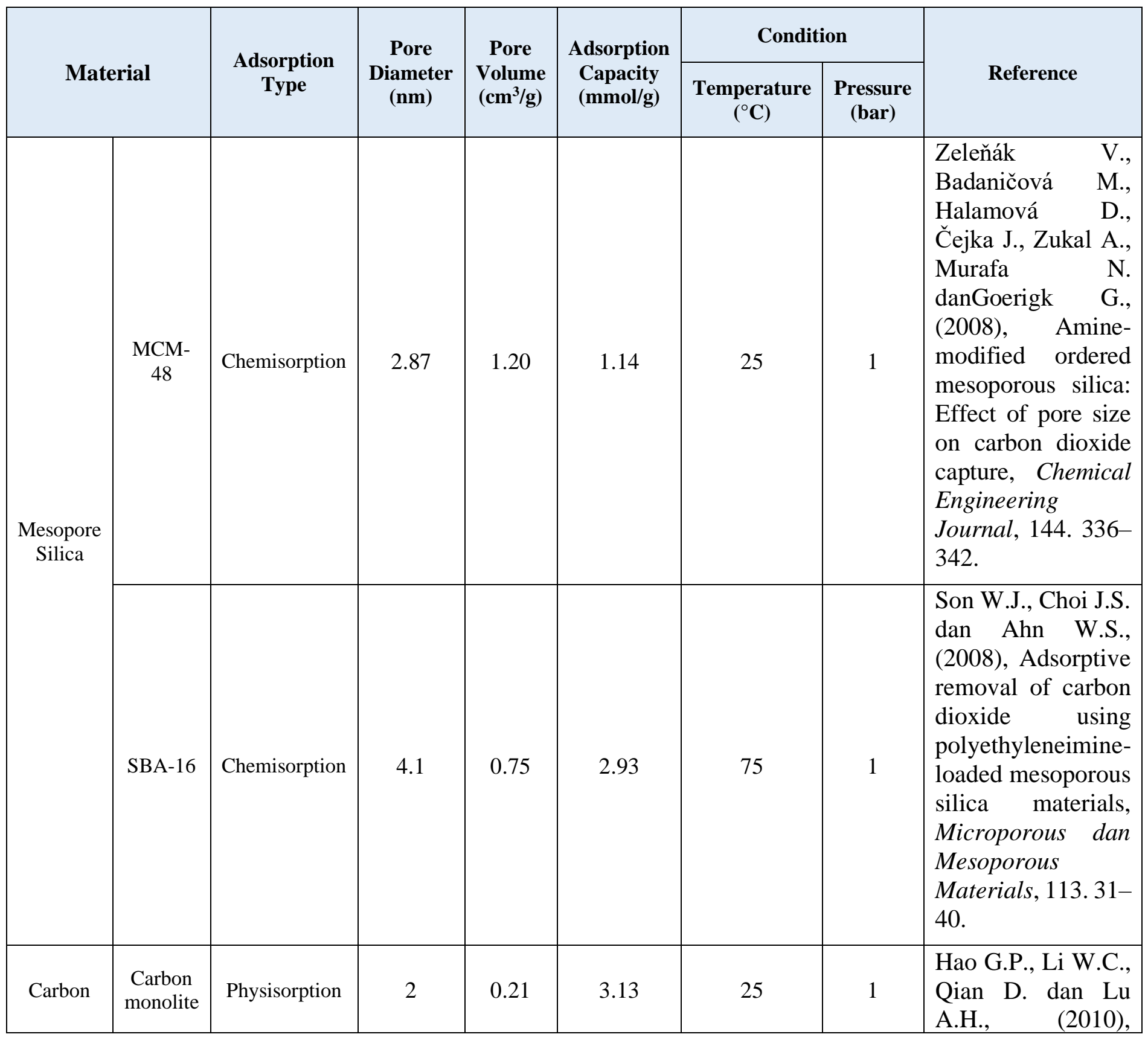




\begin{tabular}{|c|c|c|c|c|c|c|c|c|}
\hline \multirow{2}{*}{\multicolumn{2}{|c|}{ Material }} & \multirow{3}{*}{$\begin{array}{c}\text { Adsorption } \\
\text { Type }\end{array}$} & \multirow{3}{*}{$\begin{array}{c}\text { Pore } \\
\text { Diameter } \\
(\mathbf{n m})\end{array}$} & \multirow{3}{*}{$\begin{array}{c}\text { Pore } \\
\text { Volume } \\
\left(\mathrm{cm}^{3} / \mathrm{g}\right)\end{array}$} & \multirow{3}{*}{$\begin{array}{c}\text { Adsorption } \\
\text { Capacity } \\
\text { (mmol/g) }\end{array}$} & \multicolumn{2}{|c|}{ Condition } & \multirow{2}{*}{ Reference } \\
\hline & & & & & & Temperature & Pressure & \\
\hline & & & & & & & & $\begin{array}{l}\text { Rapid Synthesis of } \\
\text { Nitrogen-Doped } \\
\text { Porous Carbon } \\
\text { Monolith for } \mathrm{CO}_{2} \\
\text { Capture, } \mathrm{Adv} \text {. } \\
\text { Material, 22. } 853- \\
\text { 857. }\end{array}$ \\
\hline \multirow{2}{*}{ Zeolite } & $\begin{array}{c}\text { Zeolite } \\
13 X\end{array}$ & Physisorption & 0.40 & 0.27 & 6.00 & 25 & 15 & $\begin{array}{l}\text { Siriwardane R., } \\
\text { Shen M., Fisher E., } \\
\text { Poston } \\
\text { danShamsi A, } \\
\text { (2001), Adsorption } \\
\text { dan desorption of } \\
\mathrm{CO}_{2} \text { on solid } \\
\text { sorbents, Journal } \\
\text { of Energy \& } \\
\text { Environmental } \\
\text { Research,1. 19-22. }\end{array}$ \\
\hline & $\begin{array}{c}\text { Zeolite } \\
\text { NaA }\end{array}$ & Physisorption & 0.66 & 0.30 & 4.21 & 0 & 1 & $\begin{array}{l}\text { Cheung, O. dkk., } \\
(2013) \text {. } \\
\text { "Adsorption } \\
\text { kinetics for } \mathrm{CO}_{2} \text { on } \\
\text { highly selective } \\
\text { zeolites NaKA and } \\
\text { nano-NaKA". } \\
\text { Ahallied Energy, } \\
112 \text {, hal. 1326- } \\
1336 \text {. }\end{array}$ \\
\hline \multirow[b]{2}{*}{ MOF } & $\begin{array}{c}\text { MOF- } \\
177\end{array}$ & Chemisorption & 0.43 & 1.59 & 33.50 & 25 & 32 & \multirow{2}{*}{$\begin{array}{l}\text { Millward, A.R. } \\
\text { danYaghi, O.M., } \\
(2005), \\
\text { "Metal-Organic } \\
\text { Frameworks with } \\
\text { Exceptionally High } \\
\text { Capacity for } \\
\text { Storage of Carbon } \\
\text { Dioxide at Room } \\
\text { Temperature", J. } \\
\text { Am. Chem. Soc., } \\
\text { 127. 17998-17999. }\end{array}$} \\
\hline & MOF-5 & Chemisorption & 0.59 & 0.31 & 2.10 & 25 & 1 & \\
\hline & & & & & & & & \\
\hline
\end{tabular}




\begin{tabular}{|c|c|c|c|c|c|c|c|c|}
\hline \multirow{2}{*}{\multicolumn{2}{|c|}{ Material }} & \multirow{3}{*}{$\begin{array}{c}\begin{array}{c}\text { Adsorption } \\
\text { Type }\end{array} \\
\\
\text { Chemisorption }\end{array}$} & \multirow{3}{*}{$\begin{array}{c}\begin{array}{c}\text { Pore } \\
\text { Diameter } \\
(\mathbf{n m})\end{array} \\
\\
\\
0.43\end{array}$} & \multirow{3}{*}{$\begin{array}{c}\begin{array}{c}\text { Pore } \\
\text { Volume } \\
\left(\mathrm{cm}^{3} / \mathrm{g}\right)\end{array} \\
\\
\\
1.55\end{array}$} & \multirow{3}{*}{$\begin{array}{c}\begin{array}{c}\text { Adsorption } \\
\text { Capacity } \\
\text { (mmol/g) }\end{array} \\
\\
27.00\end{array}$} & \multicolumn{2}{|c|}{ Condition } & \multirow[b]{2}{*}{ Reference } \\
\hline & & & & & & Temperature & Pressure & \\
\hline $\mathrm{COF}$ & $\begin{array}{c}\text { COF- } \\
102\end{array}$ & & & & & 25 & 55 & $\begin{array}{l}\text { Furukawa, H. } \\
\text { danYaghi, O. M., } \\
\text { (2009), Storage of } \\
\text { hydrogen, } \\
\text { methane, dan } \\
\text { carbon dioxide in } \\
\text { highly porous } \\
\text { covalent organic } \\
\text { frameworks for } \\
\text { clean energy } \\
\text { applications, } \\
\text { Journal of the } \\
\text { American } \\
\text { Chemical Society, } \\
\text { 131. 8875-8883. }\end{array}$ \\
\hline
\end{tabular}

Mesopore silica material, MOF, and COF have a type of chemical adsorption, while carbon and zeolites are physical adsorption. Chemical adsorption requires relatively high pressure to adsorp $\mathrm{CO}_{2}$ gas in MOF and COF materials. However, when chemical adsorption uses relatively low pressures in mesopore silica material, the adsorption ability will decrease. In addition, chemical adsorption requires high energy during desorption because there is a chemical bond between carbon dioxide and material sorbent, it could difficult to release from $\mathrm{CO}_{2}$ capture material (Miller, 2011). In the physical adsorption on carbon and zeolite materials, the temperature and pressure used are relatively low and the carbon dioxide adsorbed is also more abundant than mesopore silica. Physical adsorption is reversible and tends to require low energy during desorption.

Besides the type of adsorption, another parameter that affects the adsorption of carbon dioxide is the pore diameter. Based on Table 4, mesopore silica has a larger pore diameter than zeolite, carbon, MOF and COF material. Carbon dioxide has a kinetic diameter of $3.3 \AA(0.33$ $\mathrm{nm})^{[21]}$. The materials which suitable for $\mathrm{CO}_{2}$ adsorption are those that have a diameter between $0.4 \mathrm{~nm}-2 \mathrm{~nm}$, and will be optimal if adsorbed by a material with a pore diameter of 0.4-0.6 $\mathrm{nm}$. For this reason, zeolite, carbon, MOF and COF materials have a pore diameter suitable for $\mathrm{CO}_{2}$ adsorption.

Another thing of affects $\mathrm{CO}_{2}$ adsorption is the adsorption capacity. Materials that have a large adsorption capacity for $\mathrm{CO}_{2}$ are carbon, zeolite, $\mathrm{MOF}$, and $\mathrm{COF}$. $\mathrm{CO}_{2}$ adsorption capacity on the MOF and COF greater than on the carbon and zeolites, nevertheless COF and MOF are chemical adsorption where the adsorption conditions require high pressure between 30 - 50 bar. While carbon and zeolite only require 1 bar of pressure and room temperature. It causes zeolite and carbon more to be developed as $\mathrm{CO}_{2}$ capture material. Several studies using zeolite and carbon for $\mathrm{CO}_{2}$ Adsorption are shown in Table 5 and Table 6. 
Tabel 5. Carbon Materials for $\mathrm{CO}_{2}$ Capture

\begin{tabular}{|c|c|c|c|c|}
\hline \multirow{2}{*}{$\begin{array}{l}\text { Carbon } \\
\text { Material }\end{array}$} & \multicolumn{2}{|c|}{ Adsorption Condition } & \multirow{2}{*}{$\begin{array}{c}\mathrm{CO}_{2} \\
\text { Adsorption } \\
\text { Capacity } \\
(\% . w t)\end{array}$} & \multirow{2}{*}{ Reference } \\
\hline & $\begin{array}{c}\text { Temperature } \\
\left({ }^{\circ} \mathbf{C}\right)\end{array}$ & Pressure & & \\
\hline \multirow[b]{2}{*}{$\begin{array}{l}\text { Mesopore } \\
\text { Carbon }\end{array}$} & 16 & $100 \mathrm{KPa}$ & 1.5 & \multirow{2}{*}{$\begin{array}{l}\text { Saha D., Deng S.G., } \\
(2010) \text {, Adsorption } \\
\text { equilibrium and } \\
\text { kinetics of } \mathrm{CO}_{2}, \mathrm{CH}_{4} \text {, } \\
\mathrm{N}_{2} \mathrm{O} \text {, and } \mathrm{NH}_{3} \text { on } \\
\text { ordered mesoporous } \\
\text { carbon, J. Colloid } \\
\text { Interface Sci. } 345 . \\
402-409\end{array}$} \\
\hline & 16 & $1000 \mathrm{KPa}$ & 3 & \\
\hline $\begin{array}{l}\text { Micropore } \\
\text { Carbon }\end{array}$ & 16 & $100 \mathrm{KPa}$ & 4 & $\begin{array}{l}\text { Liu Y., Yang Y., } \\
\text { Zhou Y., Zhang Y., } \\
\text { Gao M. and Pan H. } \\
\text { (2012) Hydrogen } \\
\text { storage properties } \\
\text { and mechanisms of } \\
\text { the Mg(BH4)2- } \\
\text { NaAlH4 system. } \\
\text { International Journal } \\
\text { of Hydrogen Energy } \\
\text { 37. 17137-17145 }\end{array}$ \\
\hline \multirow{4}{*}{$\begin{array}{l}\text { Activated } \\
\text { Carbon }\end{array}$} & 16 & $100 \mathrm{KPa}$ & 2.27 & \multirow{4}{*}{$\begin{array}{l}\text { Z.J. Zhang, W. } \\
\text { Zhang, X. Chen, Q.B. } \\
\text { Xia, Z. Li, (2010) } \\
\text { Adsorption of } \mathrm{CO}_{2} \text { on } \\
\text { zeolite 13X and } \\
\text { activated carbon with } \\
\text { higher surface area, } \\
\text { Sep. Sci. Technol. } 45 . \\
\text { 710-719. }\end{array}$} \\
\hline & 16 & $3000 \mathrm{KPa}$ & 21.29 & \\
\hline & 16 & $0.1-0.4$ bar & $0.6-1.5$ & \\
\hline & 55 & $0.1-0.4$ bar & $0.25-0.8$ & \\
\hline $\begin{array}{c}\text { Activated } \\
\text { Carbon ZTC-Y }\end{array}$ & 30 & $1 \mathrm{bar}$ & 2.72 & \multirow[b]{2}{*}{ 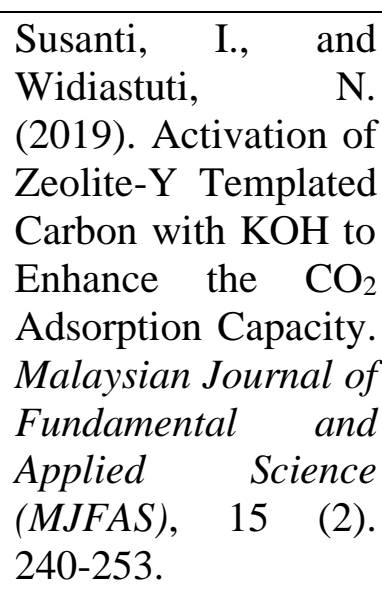 } \\
\hline Carbon ZTC-Y & 30 & 1 bar & 1.07 & \\
\hline
\end{tabular}


Tabel 6. Zeolite Materials for $\mathrm{CO}_{2}$ Capture

\begin{tabular}{|c|c|c|c|c|}
\hline \multirow{2}{*}{$\begin{array}{l}\text { Zeolite } \\
\text { Type }\end{array}$} & \multicolumn{2}{|c|}{ Adsorption Condition } & \multirow{2}{*}{$\begin{array}{l}\mathrm{CO}_{2} \text { Adsorption } \\
\text { Capacity }(\% . w t)\end{array}$} & \multirow[b]{2}{*}{ Reference } \\
\hline & $\begin{array}{c}\text { Temperature } \\
\left({ }^{\circ} \mathbf{C}\right)\end{array}$ & $\begin{array}{l}\text { Pressure } \\
\text { (bar) }\end{array}$ & & \\
\hline \multirow{3}{*}{$\mathrm{NaX}$} & 16 & $0.1-0.4$ & $2.8-2.9$ & \multirow{2}{*}{$\begin{array}{l}\text { Cavenati, S., Grande, C. A. } \\
\text { \& Rodrigues, A. E., (2004). } \\
\text { Adsorption Equilibrium of } \\
\text { Methane, Carbon Dioxide, } \\
\text { and Nitrogen on Zeolite 13X } \\
\text { at High Pressure. Journal of } \\
\text { Chemical Engineering, } 49 . \\
\text { 1095-1101. }\end{array}$} \\
\hline & 50 & $0.1-0.4$ & $1.43-2.49$ & \\
\hline & 25 & 1 & 4.00 & $\begin{array}{l}\text { Walton K. S., Abney M. B. } \\
\text { dan Douglas LeVan M., } \\
(2006), \mathrm{CO}_{2} \text { adsorption in Y } \\
\text { dan X zeolites modified by } \\
\text { alkali metal cation exchange, } \\
\text { Microporous dan } \\
\text { Mesoporous Materials, } 91 . \\
\text { 78-84. }\end{array}$ \\
\hline \multirow{2}{*}{$\mathrm{NaY}$} & 50 & $0.1-0.4$ & $0.45-1.17$ & $\begin{array}{l}\text { Cavenati, S., Grande, C. A. } \\
\text { \& Rodrigues, A. E., (2004). } \\
\text { Adsorption Equilibrium of } \\
\text { Methane, Carbon Dioxide, } \\
\text { and Nitrogen on Zeolite 13X } \\
\text { at High Pressure. Journal of } \\
\text { Chemical Engineering, } 49 . \\
\text { 1095-1101. }\end{array}$ \\
\hline & 30 & 1 & 4.00 & $\begin{array}{l}\text { Yu L., Gong J., Zeng C. dan } \\
\text { Zhang L., (2013), Synthesis } \\
\text { of binderless zeolite X } \\
\text { microspheres dan their } \mathrm{CO}_{2} \\
\text { adsorption properties, } \\
\text { Separation dan Purification } \\
\text { Technology, 118. 188-195. }\end{array}$ \\
\hline $\mathrm{NaA}$ & 30 & 1 & 2.70 & $\begin{array}{l}\text { Zukal A., Arean C. O., } \\
\text { Delgado M. R., Nachtigall P., } \\
\text { Pulido A., Mayerová J. } \\
\text { danČejka J., (2011), } \\
\text { Combined volumetric, } \\
\text { infrared spectroscopic dan } \\
\text { theoretical investigation of } \\
\mathrm{CO}_{2} \text { adsorption on Na-A } \\
\text { zeolite, } \\
\text { MicroporousdanMesoporous } \\
\text { Materials, 146. 97-105. }\end{array}$ \\
\hline
\end{tabular}




\begin{tabular}{|c|c|c|c|c|}
\hline \multirow{3}{*}{$\begin{array}{l}\text { Zeolite } \\
\text { Type }\end{array}$} & \multicolumn{2}{|c|}{ Adsorption Condition } & \multirow{2}{*}{$\begin{array}{l}\mathrm{CO}_{2} \text { Adsorption } \\
\text { Capacity (\%.wt) }\end{array}$} & \multirow[b]{2}{*}{ Reference } \\
\hline & $\begin{array}{c}\text { Temperature } \\
\left({ }^{\circ} \mathrm{C}\right)\end{array}$ & $\begin{array}{l}\text { Pressure } \\
\text { (bar) }\end{array}$ & & \\
\hline & 0 & 1 & 4.21 & $\begin{array}{l}\text { Cheung, O. dkk., (2013). } \\
\text { "Adsorption kinetics for } \mathrm{CO}_{2} \\
\text { on highly selective zeolites } \\
\text { NaKA and nano-NaKA". } \\
\text { Ahallied Energy, 112, hal. } \\
\text { 1326-1336. }\end{array}$ \\
\hline $\mathrm{NaKA}$ & 0 & 1 & 3.77 & $\begin{array}{l}\text { Cheung, O. dkk., (2013). } \\
\text { "Adsorption kinetics for } \mathrm{CO}_{2} \\
\text { on highly selective zeolites } \\
\text { NaKA and nano-NaKA". } \\
\text { Ahallied Energy, 112, hal. } \\
\text { 1326-1336. }\end{array}$ \\
\hline $\mathrm{CaX}$ & 25 & 1 & 17.55 & $\begin{array}{l}\text { Yu L., Gong J., Zeng C. dan } \\
\text { Zhang L., (2013), Synthesis } \\
\text { of binderless zeolite X } \\
\text { microspheres dan their } \mathrm{CO}_{2} \\
\text { adsorption properties, } \\
\text { Separation dan Purification } \\
\text { Technology, 118. 188-195. }\end{array}$ \\
\hline \multirow{2}{*}{$13 \mathrm{X}$} & 25 & 1 & 4.50 & $\begin{array}{l}\text { Siriwardane R., Shen M., } \\
\text { Fisher E., Poston J. } \\
\text { danShamsi A, (2001), } \\
\text { Adsorption dan desorption of } \\
\mathrm{CO}_{2} \text { on solid sorbents, } \\
\text { Journal of Energy \& } \\
\text { Environmental Research,1. } \\
\text { 19-22. }\end{array}$ \\
\hline & 25 & 15 & 6.00 & $\begin{array}{l}\text { McEwen, J., Hayman, J.-D. } \\
\text { \& Yazaydin, A. O., (2013). } \\
\text { A comparative study of } \mathrm{CO}_{2} \text {, } \\
\mathrm{CH} 4 \text { and N2 adsorption in } \\
\text { ZIF-8, Zeolit-13X and BPL } \\
\text { activated carbon. Chemical } \\
\text { Physics, 412. 72-76. }\end{array}$ \\
\hline
\end{tabular}

\section{KESIMPULAN (CONCLUSION)}

Based on the description of technology and material for $\mathrm{CO}_{2}$ adsorption above, it can be concluded that $\mathrm{CO}_{2}$ adsorption can be carried out through absorption, adsorption, membrane and cryogenic technologies. From these techniques, the adsorption method is considered more efficient and can be applied with various porous materials such as mesoporous silica, zeolite, carbon, MOF and COF. Porous material has each advantages and disadvantages. Zeolites and carbon are more interesting to develop due to zeolites have good porosity but a small surface area and carbon has a large surface area but irregular porosity. There have been several studies 
that have combined carbon and zeolite materials into zeolite-templated carbon with synthetic zeolite and resulted in increased adsorption capacity.

\section{SARAN (SUGGESTIONS)}

One proposal that can be done on further research in the development of adsoption materials is combine zeolite and carbon into zeolite/carbon composite. Zeolite/carbon composite materials can be synthesized with basic materials from nature, such as rice husks, bagasse and others. By utilizing natural waste as a basic material for making composites material, the cost of making zeolite/carbon composites material becomes cheaper and the waste of natural materials can be increased in value.

\section{UCAPAN TERIMA KASIH (ACKNOWLEDGMENT)}

Author would like to Universitas Islam Lamongan, East Java, Indonesia for providing research facility and help for this paper

\section{DAFTAR PUSTAKA (REFERENCES)}

[1] Oh, T.H. (2010). Carbon Capture and Storage Potential in Coal-fired Plant in Malaysiaa Review Renewable Sustainable Energy Rev. 14. 2697-2709.

[2] Li, J.-R.dkk., (2011). Carbon dioxide capture-related gas adsorption and separation in metal-organic frameworks. Coordination Chemistry Reviews, 255. 1791-1823.

[3] Yang, H. dkk., (2008). Progress in carbon dioxide and capture: A review. Journal of Enviromental Sciences, 20. 14-27.

[4] Figueroa J. D., Fout T., Plasynski S., Mc. Ilvried H. dan Srivastava R. D., (2008), Advances in $\mathrm{CO}_{2}$ capture technology, The U.S. Department of Energy's Carbon Sequestration Program, International Journal of Greenhouse Gas Control, 21. 9-20.

[5] Kintisch, E., (2014). Can Sucking $\mathrm{CO}_{2}$ Out of the Atmosphere Really Work, Columbia: MIT Technology Review.

[6] Bielicki, J. M. dkk., (2014). Causes and financial consequences of geologic $\mathrm{CO}_{2}$ storage reservoir leakage and interference with other subsurface resources. International Journal of Greenhouse Gas Control, 20. 272-284.

[7] Bennaucer, K., Gielen, D., Kerr, T. \& Tam, C., (2008). $\mathrm{CO}_{2}$ Capture and Storage. International Energy Agency, p. 15.

[8] Li, J.-R.dkk., (2011). Carbon dioxide capture-related gas adsorption and separation in metal-organic frameworks. Coordination Chemistry Reviews, 255. 1791-1823.

[9] Miller, B.G., (2011) $\mathrm{CO}_{2}$ Capture dan Storage, In Clean Coal Engineering Technology, Butterworth-Heinemann, Boston, 483-511.

[10] Pirngruber G. D., Guillou F., Gomez A. dan Clausse M., (2013), A theoretical analysis of the energy consumption of post-combustion $\mathrm{CO}_{2}$ capture processes by temperature swing adsorption using solid sorbents", International Journal of Greenhouse Gas Control, 14. 74-83.

[11] Smith, N. dkk., (2013). Performance and Costs of $\mathrm{CO}_{2}$ Capture at Gas Fired Power Plants. Energy Procedia, 37. 2443-2452.

[12] Cheung, O. dkk., (2013). "Adsorption kinetics for $\mathrm{CO}_{2}$ on highly selective zeolites NaKA and nano-NaKA". Ahallied Energy, 112, hal. 1326-1336.

[13] Pham T. D., Xiong R., Sdanler S. I. dan Lobo R. F., (2014) Experimental dan computational studies on the adsorption of $\mathrm{CO}_{2}$ dan $\mathrm{N}_{2}$ on pure silica zeolites. Microporous dan Mesoporous Materials, 185. 157-166. 
[14] Kazama, S. dan Haraya, K., (2013), Optimization of $\mathrm{CO}_{2}$ concentration captured by membrane technology - Possibility of reduction in $\mathrm{CO}_{2}$ capture energy dan cost, Energy Procedia, 37. 969-975.

[15] Son W.J., Choi J.S. dan Ahn W.S., (2008), Adsorptive removal of carbon dioxide using polyethyleneimine-loaded mesoporous silica materials, Microporous dan Mesoporous Materials, 113. 31-40.

[16] Zeleňák V., Badaničová M., Halamová D., Čejka J., Zukal A., Murafa N. danGoerigk G., (2008), Amine-modified ordered mesoporous silica: Effect of pore size on carbon dioxide capture, Chemical Engineering Journal, 144. 336-342.

[17] Hao G.P., Li W.C., Qian D. dan Lu A.H., (2010), Rapid Synthesis of Nitrogen-Doped Porous Carbon Monolith for $\mathrm{CO}_{2}$ Capture, Adv. Material, 22. 853-857.

[18] Siriwardane R., Shen M., Fisher E., Poston J. danShamsi A,. (2001), Adsorption dan desorption of $\mathrm{CO}_{2}$ on solid sorbents, Journal of Energy \& Environmental Research, 1. $19-22$.

[19] Millward, A.R. danYaghi, O.M., (2005), "Metal-Organic Frameworks with Exceptionally High Capacity for Storage of Carbon Dioxide at Room Temperature", $J$. Am. Chem. Soc., 127. 17998-17999.

[20] Furukawa, H. danYaghi, O. M., (2009), Storage of hydrogen, methane, dan carbon dioxide in highly porous covalent organic frameworks for clean energy applications, Journal of the American Chemical Society, 131. 8875-8883.

[21] Susanti, Indri. (2019). Polysulfone Membrane with Zeolite Filler for $\mathrm{CO}_{2} / \mathrm{CH}_{4} \mathrm{Gas}$ Separation: a Review. Science Education and Application Journal (SEAJ). 1 (1). 1016.

[22] Saha D., Deng S.G., (2010), Adsorption equilibrium and kinetics of $\mathrm{CO}_{2}, \mathrm{CH}_{4}, \mathrm{~N}_{2} \mathrm{O}$, and $\mathrm{NH}_{3}$ on ordered mesoporous carbon, J. Colloid Interface Sci. 345. 402-409.

[23] Z.J. Zhang, W. Zhang, X. Chen, Q.B. Xia, Z. Li, (2010). Adsorption of $\mathrm{CO}_{2}$ on zeolite $13 X$ and activated carbon with higher surface area, Sep. Sci. Technol. 45. 710-719.

[24] Liu Y., Yang Y., Zhou Y., Zhang Y., Gao M. and Pan H. (2012) Hydrogen storage properties and mechanisms of the $\mathrm{Mg}(\mathrm{BH} 4) 2-\mathrm{NaAlH} 4$ system. International Journal of Hydrogen Energy 37. 17137-17145

[25] Susanti, I., and Widiastuti, N. (2019). Activation of Zeolite-Y Templated Carbon with $\mathrm{KOH}$ to Enhance the $\mathrm{CO}_{2}$ Adsorption Capacity. Malaysian Journal of Fundamental and Applied Science (MJFAS), 15 (2). 240-253.

[26] Cavenati, S., Grande, C. A. \& Rodrigues, A. E., (2004). Adsorption Equilibrium of Methane, Carbon Dioxide, and Nitrogen on Zeolite 13X at High Pressure. Journal of Chemical Engineering, 49. 1095-1101.

[27] Walton K. S., Abney M. B. dan Douglas LeVan M., (2006), $\mathrm{CO}_{2}$ adsorption in Y dan $\mathrm{X}$ zeolites modified by alkali metal cation exchange, Microporous dan Mesoporous Materials, 91. 78-84.

[28] Yu L., Gong J., Zeng C. dan Zhang L., (2013), Synthesis of binderless zeolite X microspheres dan their $\mathrm{CO}_{2}$ adsorption properties, Separation dan Purification Technology, 118. 188-195.

[29] Zukal A., Arean C. O., Delgado M. R., Nachtigall P., Pulido A., Mayerová J. danČejka J., (2011), Combined volumetric, infrared spectroscopic dan theoretical investigation of $\mathrm{CO}_{2}$ adsorption on Na-A zeolite, MicroporousdanMesoporous Materials, 146. 97-105.

[30] McEwen, J., Hayman, J.-D. \& Yazaydin, A. O., (2013). A comparative study of $\mathrm{CO}_{2}$, CH4 and N2 adsorption in ZIF-8, Zeolit-13X and BPL activated carbon. Chemical Physics, 412. 72-76 